\title{
Continuous selections from the Pareto correspondence and non-manipulability in exchange economies
}

\author{
Biung-Ghi Ju* \\ University of Kansas, Department of Economics \\ January 8, 2003 (Revision May 2003)
}

\begin{abstract}
In $n$-agent exchange economies, we show that all efficient and continuous rules are "diagonally dictatorial" over the restricted domain of linear preferences and, in the 2-good case, over the domain of homothetic preferences. The diagonal dictator receives the entire endowment whenever all agents have an identical preference. We show that (fully) dictatorial rules are the only rules satisfying, in addition, veto-proofness, the requirement that if truth-telling ever leads to the worst outcome for an agent, he shouldn't be able to escape it, by misrepresenting his preference. The same conclusion holds replacing veto-proofness with stronger notions of non-manipulability, veto-proofness* (no one can escape from the worst outcome or switch to the best outcome), weak strategy-proofness (no one can increase his bundle), and strategy-proofness. We extend these results to any larger domain imposing non-bossiness (no one can affect others' bundles without affecting his own).
\end{abstract}

Keywords: Efficiency, continuity, veto-proofness, strategy-proofness, exchange economy.

JEL Classification Numbers: C70, D70.

*Department of Economics, 1300 Sunnyside Avenue, Summerfield Hall, University of Kansas, KS 66045, USA. E-mail: bgju@ku . edu. Tel.: (785) 864-2860. Fax.:(785) 864-5270. I am grateful to William Thomson for helpful comments and suggestions. I also thank John Duggan, Lars Ehlers, Sidartha Gordon, James Schummer, and an anonymous referee for comments. All remaining errors are mine. 


\section{Introduction}

In exchange economies, an allocation rule, or simply a rule, associates with each profile of preferences a single feasible allocation. An allocation is (Pareto) efficient if no one can be made better off without anyone else being made worse off. The Pareto correspondence maps each preference profile into the set of all efficient allocations. An efficient rule is a selection from the Pareto correspondence. We study continuous selections from the Pareto correspondence, or efficient and continuous rules, and, in particular, these rules satisfying the following notions of non-manipulability.

Strategy-proofness is the requirement that no one can benefit by misrepresenting his preference, independently of others' representations (Gibbard, 1973, Satterthwaite, 1975). We introduce three weaker notions of non-manipulability, which pertain to three cases of partially strategic agents. The first case is that each agent behaves cooperatively representing his preference truthfully, unless he is treated worst. Veto-proofness is the requirement that if truth-telling ever leads to the worst outcome for an agent, he shouldn't be able to escape it, by misrepresenting his preference. A stronger notion, veto-proofness*, pertains to the case that each agent represents his preference truthfully, unless he can escape from the worst outcome or can switch to the best outcome. The last notion, weak strategy-proofness, pertains to the case that each agent represents his preference truthfully, unless he can increase his bundle in terms of vector dominance relation.

We show that over the restricted domain of "linear preferences" (preferences with linear utility function), every efficient and continuous rule is diagonally dictatorial, that is, there exists an agent who receives the social endowment, whenever all agents have the same preference. Therefore, for any domain including the linear domain, there exists no efficient and continuous rule satisfying any one of the following standard equity criteria, equal treatment of equals (any two agents with the same preference should be treated the same), no-envy (Foley, 1967; no one should prefer any of others' bundles to his own), etc. ${ }^{1}$ We next show that over the linear domain, a rule is efficient, continuous, and veto-proof if and only if it is dictatorial, that is, there exists an agent who always receives the social endowment, leaving nothing for anyone else. The same result holds replacing veto-proofness with any one of veto-proofness*, weak strategy-proofness, and strategy-proofness. We extend this result over the linear domain to any larger domain with the additional requirement of non-bossiness (no one can affect others'

\footnotetext{
${ }^{1}$ See Thomson (1995) for an extensive treatment of fairness in economic environments.
} 
bundle without affecting his own bundle) introduced by Satterthwaite and Sonnenschein (1981). Given any domain including the linear domain, we show that a rule is efficient, continuous, veto-proof* (or weakly strategy-proof), and non-bossy if and only if it is dictatorial. Since all linear preferences are "homothetic", this result applies to the domain of homothetic preferences, or the homothetic domain, which is widely considered in various applications (Chipman, 1974, and Chipman and Moore, 1973). In the 2-good case, we establish the following two stronger results over the homothetic domain: we show that every efficient and continuous rule is diagonally dictatorial and that a rule is efficient, continuous, and veto-proof if and only if it is dictatorial. The second result holds replacing veto-proofness with veto-proofness* or weak strategy-proofness or strategy-proofness.

For the unrestricted domain of abstract social choice, the Gibbard-Satterthwaite Theorem (Gibbard, 1973, Satterthwaite, 1975) states that if a rule is strategy-proof and "onto", then it is dictatorial. ${ }^{2}$ Since in economic applications, preferences are subject to a variety of restrictions, the theorem does not apply. ${ }^{3}$ However a number of studies have brought out similar difficulties in satisfying efficiency and strategy-proofness in standard exchange economies. ${ }^{4}$ In particular, for the 2-agent case, under "classical" assumptions on preferences, Zhou (1991) shows that a rule is efficient and strategy-proof if and only if it is dictatorial. Schummer (1997) strengthens this result by establishing it over the two restricted domains of homothetic preferences and linear preferences, respectively. Ju (2002) identifies general domain conditions leading to this impossibility result.

There are well-known difficulties in extending this negative result in the 2agent case to the $n$-agent case: see Zhou (1991) and Kato and Ohseto (2001) for conjectures in the $n$-agent case. We contribute to this line of research by showing that over each of the two restricted domains, the linear domain and the 2-good homothetic domain, when continuity is required additionally, the negative result extends to the $n$-agent case.

In the $n$-agent case, important contributions have been made in the three recent works by Serizawa (2000a), Serizawa (2000b), and Serizawa and Wey-

\footnotetext{
${ }^{2} \mathrm{~A}$ rule is onto if its range is equal to the set of all social alternatives. In the abstract social choice model, if a rule is Pareto efficient, then it is onto. Moreover, as is well-known (see Mas-Colell, Whinston, and Green, 1995), if a rule is onto and strategy-proof, then it is Pareto efficient.

${ }^{3}$ In exchange economies, preferences are assumed to satisfy "no-consumption-externality" (individuals are not affected by others' consumption) and "monotonicity".

${ }^{4}$ References are Hurwicz (1972), Dasgupta, Hammond, and Maskin (1979), Hurwicz and Walker (1990), Zhou (1991a), Schummer (1997), and Serizawa (1998). See also the extensive surveys by Sprumont (1995a), Barberà (2001), and Thomson (2001)
} 
mark (2002). Serizawa (2000a) shows that there is no rule satisfying efficiency, individual rationality, and strategy-proofness. Replacing individual rationality with a much weaker axiom, called "minimum consumption guarantee", Serizawa and Weymark (2002) establish an even stronger impossibility. Serizawa (2000b) does not impose continuity but considers a stronger non-manipulability notion, "pairwise strategy-proofness" associated with preference misrepresentation by groups consisting of at most two agents.

Continuity is desirable for the following practical reason. In order to make a choice, we need information about preferences. However, even if people are willing to reveal their own true preferences, for a number of reasons, they may not be known accurately. If a rule is not continuous, the choice it makes may be vulnerable to such inaccuracy. ${ }^{5}$

In the 2-agent and 2-good case, Sprumont (1995b) characterizes continuous and strategy-proof rules over the same homothetic domain as ours. He shows that any continuous and strategy-proof rule has the following dictatorial feature: there exists an agent and an exogenously determined strictly convex subset of consumption space such that the rule picks the best point for the agent in the set. Our results show that in the n-agent case, even if strategy-proofness is replaced with efficiency, we still have "diagonal dictatorship", which implies strong violation of most standard equity criteria.

Continuity and strategy-proofness are studied also by Satterthwaite and Sonnenschein (1981), yet on the domain consisting of only strictly convex preferences. They impose in addition, "continuous differentiability" and non-bossiness. The domain of production economies with convex technologies is one of the domains they consider, and this domain includes exchange economies. Their result for this domain implies that there exists no rule that is efficient, continuously differentiable, strategy-proof, and non-bossy. However they do not study consequences of dropping non-bossiness or weakening continuous differentiability to continuity or weakening strategy-proofness. ${ }^{6}$

\footnotetext{
${ }^{5}$ In the implementation literature, one of the desirable properties of a game form is continuity of its outcome function (See Postlewaite and Wettstein, 1989). Continuity guarantees the robustness of equilibrium to small misspecifications of strategies. Outcome functions are often closely related with rules they implement. In particular, in the direct revelation mechanism, the rule coincides with the outcome function.

${ }^{6}$ In various models, central strategy-proof rules are not differentiable. For example, in public good economies with single-peaked preference considered by Moulin (1980), the "generalized Condorcet-winner rules", which are the only rules satisfying Pareto efficiency, anonymity, and strategy-proofness, are not differentiable. In private good economies with single-peaked preferences considered by Sprumont (1991), the "uniform rule", which is the only rule satisfying
} 
Chichilnisky (1979, 1980, 1982), Chichilnisky and Heal (1983), and Zhou (1997) study continuous "preference aggregation rule", which is a function mapping each preference profile into a social "preference". These studies exhibit difficulties of satisfying continuity together with other equity criteria such as "unanimity" (similar to our efficiency) and "anonymity" (similar to equal treatment of equals). Although their results are established for "preference aggregation rules" and in the topological social choice model, our result on diagonal dictatorship has a similar flavor to theirs.

This paper is composed of five sections. In Section 2, we define our model and basic concepts. In Section 3, we establish several useful lemmas. Our main results are in Section 4. We conclude with a few remarks in Section 5.

\section{The model and basic concepts}

Let $l$ be the number of goods, $l \geq 2$, and $\Omega \in \mathbb{R}_{+}^{l}$ be the social endowment. Let $N \equiv\{1, \cdots, n\}$ be the set of agents. Let $Z \equiv\left\{z \in \mathbb{R}_{+}^{l \cdot n}: \sum_{N} z_{i}=\Omega\right\}$ be the set of feasible allocations and $Z_{0} \equiv\left\{z_{i} \in \mathbb{R}_{+}^{l}: 0 \leqq z_{i} \leqq \Omega\right\}$ the set of possible consumption bundles for each agent.

Each agent has a preference, a complete and transitive binary relation over $\mathbb{R}_{+}^{l}$. Preferences are continuous, strictly monotonic, ${ }^{7}$, and convex. For each preference $R_{0}$, we use $P_{0}$ and $I_{0}$ to denote its strict relation and indifference relation, respectively. Let $\mathcal{R}$ be a family of admissible preferences. Since we keep the social endowment fixed, an economy can be identified by a profile of preferences in $\mathcal{R}$. An allocation rule, or simply a rule, is a function $\varphi: \mathcal{R}^{N} \rightarrow Z$ associating with each economy a single feasible allocation.

The following two restricted families of preferences are important. A preference $R_{0}$ is homothetic if for all $x, y \in \mathbb{R}_{+}^{l}$ and all $\alpha \in \mathbb{R}_{+}, x I_{0} y$ implies $\alpha x I_{0} \alpha y$. Let $\mathcal{R}_{H}$ be the class of homothetic preferences. A preference $R_{0}$ is linear if it is represented by a vector $p_{0} \in \mathbb{R}_{++}^{l}$ as follows: for all $x, y \in \mathbb{R}_{+}, x R_{0} y$ if and only if $p_{0} \cdot x \geq p_{0} \cdot y$. Let $\mathcal{R}_{L}$ be the class of linear preferences. Clearly, $\mathcal{R}_{L} \subset \mathcal{R}_{H}$. We call $\mathcal{R}_{H}^{N}$ and $\mathcal{R}_{L}^{N}$ the homothetic domain and the linear domain, respectively. Note that since preferences are strictly monotonic, for all homothetic preference, every indifference curve intersects with each axis. Thus,

Pareto efficiency, anonymity, and strategy-proofness, is not differentiable.

${ }^{7} \mathrm{~A}$ preference relation $R_{i}$ is strictly monotonic if $z_{i} \geq z_{i}^{\prime}$ implies $z_{i} P_{i} z_{i}^{\prime}$, where the vector inequality $z_{i} \geq z_{i}^{\prime}$ means that each component of $z_{i}$ is weakly larger than each component of $z_{i}^{\prime}$ and $z_{i} \neq z_{i}^{\prime}$. 
for example, the "Cobb-Douglas" preferences are not members of $\mathcal{R}_{H}$. However, this feature is not crucial for our result as explained in Section 5.

For each economy $R \in \mathcal{R}^{N}$, an allocation $z \in Z$ is (Pareto) efficient if there is no feasible allocation that makes at least one agent better off without making anyone else worse off. Let $P(R)$ be the set of all efficient allocations for $R$. We call $P(\cdot)$, the Pareto correspondence. A rule $\varphi: \mathcal{R}^{N} \rightarrow Z$ is efficient if it is a selection from the Pareto correspondence, that is, for all $R \in \mathcal{R}^{N}$, $\varphi(R) \in P(R)$.

Domain $\mathcal{R}_{H}$ is endowed with the metric $\rho: \mathcal{R}_{H} \times \mathcal{R}_{H} \rightarrow \mathbb{R}_{+}$defined as follows. For all $x, y \in \mathbb{R}^{l}$, let $\overrightarrow{x, y} \equiv\left\{x+\alpha(y-x): \alpha \in \mathbb{R}_{+}\right\}$and $\overline{x, y} \equiv\{x+\alpha(y-x): \alpha \in$ $[0,1]\}$. For all $R_{0} \in \mathcal{R}_{H}$ and all $q \in \Delta^{l-1}$, let $r\left(q, R_{0}\right) \in \mathbb{R}_{+}^{l}$ be the point of intersection of the ray, $\overrightarrow{0, q}$, and the indifference curve of $R_{0}$ through $(1, \cdots, 1)$ (note that $r\left(q, R_{0}\right)$ is well-defined because preferences are strictly monotonic). For all $R_{0}, R_{0}^{\prime} \in \mathcal{R}_{H}$, let $\rho\left(R_{0}, R_{0}^{\prime}\right) \equiv \sup _{q \in \Delta^{l-1}}\left\|r\left(q, R_{0}\right)-r\left(q, R_{0}^{\prime}\right)\right\|$. For convenience, over the subdomain $\mathcal{R}_{L}$, we use the following equivalent metric $d: \mathcal{R}_{L} \times \mathcal{R}_{L} \rightarrow \mathbb{R}_{+}$: for all $R_{i}, R_{i}^{\prime} \in \mathcal{R}_{L}$, let $p_{i} \in \Delta^{l-1}$ represent $R_{i}$ and $p_{i}^{\prime} \in \Delta^{l-1}$ represent $R_{i}^{\prime}$. Let $d\left(R_{i}, R_{i}^{\prime}\right) \equiv\left\|p_{i}-p_{i}^{\prime}\right\|$. The topologies of $\mathcal{R}_{L}^{N}$ and $\mathcal{R}_{H}^{N}$ are the product topologies corresponding to the metric topologies of $\mathcal{R}_{L}$ and $\mathcal{R}_{H}$.

In what follows, for any family of preferences, denoted by $\mathcal{R}$, when $\mathcal{R}$ contains $\mathcal{R}_{H}$ (or $\mathcal{R}_{L}$ ), we assume that $\mathcal{R}$ is endowed with a topology inducing the above metric topology in the subspace $\mathcal{R}_{H}$ (or $\mathcal{R}_{L}$, respectively) and that $\mathcal{R}^{N}$ is endowed with the product topology associated with the topology on $\mathcal{R}$. We refer readers to Kannai (1970) for a construction of topology on spaces of preferences.

Given a topology of $\mathcal{R}^{N}$, we define continuity of an allocation rule over $\mathcal{R}^{N}$ in the standard way. We study efficient and continuous rules, or continuous selections from the Pareto correspondence, which are immune to the following kinds of manipulative behavior.

Most well-known is manipulation via preference misrepresentation. A rule $\varphi: \mathcal{R}^{N} \rightarrow Z$ is strategy-proof if no one can ever benefit by misrepresenting his preference, independently of others' representations; that is, for all $i \in N$ and all $R \in \mathcal{R}^{N}$, there exists no $R_{i}^{\prime} \in \mathcal{R}$ such that $\varphi_{i}\left(R_{i}^{\prime}, R_{-i}\right) P_{i} \varphi_{i}\left(R_{i}, R_{-i}\right)$.

We next define three weaker notions of non-manipulability, which pertain to three types of partially strategic behavior of agents. First is the type in which each agent behaves cooperatively, truthfully representing his preference, except when he is treated worst. Each agent misrepresents his preference only to "veto" the worst outcome, namely, the zero bundle. In this case, the following weakening of strategy-proofness is important. A rule $\varphi: \mathcal{R}^{N} \rightarrow Z$ is veto-proof if for all 
$i \in N$ and all $R \in \mathcal{R}^{N}$ with $\varphi_{i}(R)=0$, there exists no $R_{i}^{\prime} \in \mathcal{R}$ such that $\varphi_{i}\left(R_{i}^{\prime}, R_{-i}\right) P_{i} \varphi_{i}\left(R_{i}, R_{-i}\right)$.

Second is the type in which each agent truthfully represents his preference, unless he can switch to the best outcome or escape the worst outcome, by misrepresenting his preference. Since preferences are strictly monotonic, $\Omega$ is the best outcome and 0 is the worst outcome. In this case, the following weakening of strategyproofness is important. A rule $\varphi: \mathcal{R}^{N} \rightarrow Z$ is veto-proof* ${ }^{*}$ if for all $i \in N$ and all $R \in \mathcal{R}^{N}$, there exists no $R_{i}^{\prime} \in \mathcal{R}$ such that (i) $\varphi_{i}\left(R_{i}^{\prime}, R_{-i}\right)=\Omega P_{i} \varphi_{i}(R)$ or (ii) $\varphi_{i}\left(R_{i}^{\prime}, R_{-i}\right) P_{i} 0=\varphi_{i}\left(R_{i}, R_{-i}\right)$.

Third is the type in which each agent truthfully represents his preference, unless he can increase his bundle in terms of vector dominance relation, by misrepresenting his preference. In this case, the following weakening of strategy-proofness is important. A rule $\varphi: \mathcal{R}^{N} \rightarrow Z$ is weakly strategy-proof if for all $i \in N$ and all $R \in \mathcal{R}^{N}$, there exists no $R_{i}^{\prime} \in \mathcal{R}$ such that $\varphi_{i}\left(R_{i}^{\prime}, R_{-i}\right) \geq \varphi_{i}(R)$.

Clearly, every rule satisfies veto-proofness if no allocation in its range has any zero component, that is, no one ever receives the zero bundle. Similarly, every rule satisfies veto-proofness* if no one ever receives either the zero bundle or $\Omega$. Since preferences are strictly monotonic, strategy-proofness implies weak strategy-proofness, which implies veto-proofness ${ }^{*}$, which implies veto-proofness.

Our main results show that all rules satisfying the above requirements have the following "dictatorial" feature. A rule is dictatorial if there exists an agent who always gets his most preferred bundle in $Z_{0}$. Since preferences are strictly monotonic, a rule $\varphi: \mathcal{R}^{N} \rightarrow Z$ is dictatorial if and only if there exists $i \in N$ such that for all $R \in \mathcal{R}^{N}, \varphi_{i}(R)=\Omega$. We call such an agent the dictator. A preference profile $R \in \mathcal{R}^{N}$ is diagonal if there exists $R_{0} \in \mathcal{R}$ such that $R=\left(R_{0}, \cdots, R_{0}\right)$. A rule $\varphi: \mathcal{R}^{N} \rightarrow Z$ is diagonally dictatorial if there exists an agent who receives his most preferred bundle in $Z_{0}$ at every diagonal preference profile. We call such an agent the diagonal dictator.

Every diagonally dictatorial rule violates the following standard equity criteria. A rule $\varphi: \mathcal{R}^{N} \rightarrow Z$ satisfies equal treatment of equals if for all $i, j \in N$ and all $R \in \mathcal{R}^{N}$ with $R_{i}=R_{j}=R_{0}, \varphi_{i}(R) I_{0} \varphi_{j}(R)$. It satisfies no-envy, (Foley, 1967) if for all $i, j \in N$ and all $R \in \mathcal{R}^{N}, \varphi_{i}(R) R_{i} \varphi_{j}(R)$. It meets the equal division lower bound property if for all $i \in N$ and all $R \in \mathcal{R}^{N}, \varphi_{i}(R) R_{i}$ $\Omega / n .^{8}$

\footnotetext{
${ }^{8}$ We refer readers to Thomson (1995) for an extensive treatment of these equity criterion in various economic environments including exchange economies.
} 


\section{Useful lemmas}

We will show later that every continuous and efficient rule selects allocations on the boundary of a predetermined "truncated (polyhedral) cone", whenever there exist at least two identical agents. In this section, we study implications of this property in conjunction with continuity and veto-proofness.

We use the following notation. For all $l$ linearly independent vectors $a_{1}, \cdots, a_{l} \in$ $\mathbb{R}^{l}$ and all $k \in\{1, \cdots, l\}$, let $\left\langle a_{1}, \cdots, a_{k}\right\rangle \equiv\left\{x \in \mathbb{R}^{l}: x=\lambda_{1} a_{1}+\cdots+\lambda_{k} a_{k}\right.$, for some $\left.\lambda_{1}, \cdots, \lambda_{k} \in \mathbb{R}\right\}$ be the space generated by the $k$ vectors, $a_{1}, \cdots, a_{k}$. Let $\left\langle a_{1}, \cdots, a_{k}\right\rangle_{+} \equiv\left\{x \in \mathbb{R}^{l}: x=\lambda_{1} a_{1}+\cdots+\lambda_{k} a_{k}\right.$, for some $\left.\lambda_{1}, \cdots, \lambda_{k} \in \mathbb{R}_{+}\right\}$be the set of positive linear combinations of the $k$ vectors, $a_{1}, \cdots, a_{k}$. ${ }^{9}$ Since $a_{1}$, $\cdots, a_{l}$ are linearly independent, then for all $x \in \mathbb{R}^{l}$, there exists a unique list of numbers, $\lambda_{1}, \cdots, \lambda_{l}$, such that $x=\lambda_{1} a_{1}+\cdots+\lambda_{l} a_{l}$. For each $X \subseteq \mathbb{R}^{l}$, let int $X$ be the interior of $X$ and $i n t_{\left\langle a_{1}, \cdots, a_{k}\right\rangle} X$ be the relative interior of $X$ in the subspace $\left\langle a_{1}, \cdots, a_{k}\right\rangle$. Note that $\operatorname{int}_{\left\langle a_{1}, \cdots, a_{k}\right\rangle}\left\langle a_{1}, \cdots, a_{k}\right\rangle_{+}=\left\{x \in \mathbb{R}^{l}\right.$ : for some strictly positive $\left.\lambda_{1}, \cdots, \lambda_{k} \in \mathbb{R}_{++}, x=\lambda_{1} a_{1}+\cdots+\lambda_{k} a_{k}\right\}$.

Given a subset $\mathcal{R}_{0} \subseteq \mathcal{R}$, a rule $\varphi: \mathcal{R}^{N} \rightarrow Z$ satisfies Property B over $\mathcal{R}_{0}^{N}$, if there exist $l$ linearly independent vectors, $a_{1}, \cdots, a_{l} \in \mathbb{R}_{+}^{l}$, such that

(i) $\Omega \in \operatorname{int}\left\langle a_{1}, \cdots, a_{l}\right\rangle_{+}$;

and for all $R \in \mathcal{R}_{0}^{N}$, if for all $i \in N, \varphi_{i}(R)=\lambda_{i 1} a_{1}+\cdots+\lambda_{i l} a_{l}$ for some $\lambda_{i 1}, \cdots$, $\lambda_{i l} \in \mathbb{R}$

(ii) for all $i, j \in N$ with $i \neq j$, all $k, m \in\{1, \cdots, l\}$ with $k \neq m$, and all $R \in \mathcal{R}_{0}^{N}$ with $R_{i}=R_{j}$,

$$
\lambda_{i k}=\lambda_{i m}=0, \text { or } \lambda_{j k}=\lambda_{j m}=0, \text { or }\left(\lambda_{i k}+\lambda_{j k}, \lambda_{i m}+\lambda_{j m}\right) \notin \mathbb{R}_{++}^{2} ;{ }^{10}
$$

(iii) for all $I \subseteq N$, if for all $i, j \in I, R_{i}=R_{j}$ and $\sum_{I} \varphi_{i}(R)=\Omega$, then for all $i \in I,\left(\lambda_{i 1}, \cdots, \lambda_{i l}\right) \geqq 0$ (that is, $\left.\varphi_{i}(R) \in\left\langle a_{1}, \cdots, a_{l}\right\rangle_{+}\right)$.

When a rule satisfies Property B over the entire domain, we say that the rule satisfies Property $\boldsymbol{B}$. For all $k \in\{1, \cdots, l\}$, let $e_{k}$ be the unit vector that has 1 in its $k^{\text {th }}$ component and 0 in every other component. Note that when $a_{1}, \cdots, a_{l}$ are unit vectors, parts (i) and (iii) hold trivially. In this case, part (ii) says that whenever two agents have the same preferences, at least one of them receives 0 or both of them receive some positive amounts of only one and the same good.

Property B imposes a severe restriction on the choice, when there is a group $I$ of agents who have identical preferences and who consume the entire social

\footnotetext{
${ }^{9}$ Such a set is called "polyhedral cone".

${ }^{10}$ The last part $\left(\lambda_{i k}+\lambda_{j k}, \lambda_{i m}+\lambda_{j m}\right) \notin \mathbb{R}_{++}^{2}$ is equivalent to $\left(\lambda_{i k}+\lambda_{j k}\right) a_{k}+\left(\lambda_{i m}+\lambda_{j m}\right) a_{m} \notin$ $\operatorname{int}_{\left\langle a_{k}, a_{m}\right\rangle}\left\langle a_{k}, a_{m}\right\rangle_{+}$.
} 
endowment in the aggregate. In this case, by part (ii), no two agents in $I$ can consume, in the aggregate, a bundle in the interior of $\left\langle a_{1}, \cdots, a_{l}\right\rangle_{+}$. Moreover, part (ii) imposes similar restrictions for all two dimensional subspaces. On the other hand, by part (i), the aggregate bundle $\Omega$ (consumed by agents in $I$ ) lies in the interior of $\left\langle a_{1}, \cdots, a_{l}\right\rangle_{+}$. Since by part (iii), individual bundles should lie in $\left\langle a_{1}, \cdots, a_{l}\right\rangle_{+}$, the only way to satisfy all three parts is to give a single agent in $I$ the entire endowment $\Omega$ and all others the zero bundle, as shown by the next lemma. An allocation $z \in Z$ is extreme if it is one of the following $l$ allocations, $(\Omega, 0, \cdots, 0),(0, \Omega, 0, \cdots, 0), \cdots,(0, \cdots, 0, \Omega)$.

Lemma 1. Let $\mathcal{R}_{0} \subseteq \mathcal{R}$. Assume that $\varphi: \mathcal{R}^{N} \rightarrow Z$ satisfies Property $B$ over $\mathcal{R}_{0}^{N}$. If $I \subseteq N$ and $R \in \mathcal{R}_{0}^{N}$ are such that for all $i, j \in I, R_{i}=R_{j}$ and $\sum_{i \in I} \varphi_{i}(R)=\Omega$, then $\varphi(R)$ is extreme.

Proof. Suppose that $\varphi$ satisfies Property B with respect to $l$ linearly independent vectors $a_{1}, \cdots, a_{l} \in \mathbb{R}_{+}^{l}$. Without loss of generality, we may assume $\Omega=a_{1}+$ $\cdots+a_{l}$. Let $I \subseteq N$ and $R \in \mathcal{R}_{0}^{N}$ be given as above. Let $z \equiv \varphi(R)$. By part (iii) of Property B, for all $i \in I, z_{i} \in\left\langle a_{1}, \cdots, a_{l}\right\rangle_{+}$. Then for each $i \in I$, there exist $\lambda_{i 1}, \cdots, \lambda_{i l} \geq 0$ such that $z_{i}=\lambda_{i 1} a_{1}+\cdots+\lambda_{i l} a_{l}$. If $\lambda_{i k}>1$ for some $i \in I$ and $k \in\{1, \cdots, l\}$, then $\sum_{j \in I \backslash i} z_{j}=\sum_{j \in N \backslash i}\left(\lambda_{j k} a_{k}+\sum_{m \neq k} \lambda_{j m} a_{m}\right)=\Omega-$ $z_{i}=\left(1-\lambda_{i k}\right) a_{k}+\sum_{m \neq k}\left(1-\lambda_{i m}\right) a_{m}$. Since $a_{1}, \cdots, a_{l}$ are linearly independent, $\sum_{j \in N \backslash i} \lambda_{j k}=1-\lambda_{i k}<0$, contradicting the fact that all $\lambda_{j k}$ 's are non-negative. Therefore, for all $j \in I$ and all $k \in\{1, \cdots, l\}, \lambda_{j k} \in[0,1]$.

Since $\sum_{i \in I} z_{i}=\Omega \in \operatorname{int}\left\langle a_{1}, \cdots, a_{l}\right\rangle_{+}$, there exist $i \in I$ and $k \in\{1, \cdots, l\}$ such that $\lambda_{i k}>0$. We first show $\lambda_{i k}=1$. Suppose, by contradiction, $\lambda_{i k}<1$. Then since $\sum_{j \in I} \lambda_{j k}=1$, there exists $j \in I \backslash i$ such that $\lambda_{j k}>0$. Take any $m \neq k$. By part (ii) of Property B, $\lambda_{i m}=0$. Then since $\sum_{h \in I} \lambda_{h m}=1$, there exists $h \in I \backslash i$ such that $\lambda_{h m}>0$. This contradicts part (ii) of Property B. Therefore, $\lambda_{i k}=1$.

Let $m \in\{1, \cdots, l\} \backslash k$. We only have to show $\lambda_{i m}=1$. If $\lambda_{i m}<1$, then since $\sum_{i \in I} z_{i}=\Omega$, there is $j \in I \backslash i$ such that $\lambda_{j m}>0$. Then since $\lambda_{i k}>0$, we have a contradiction to part (ii) of Property B. Therefore $\lambda_{i m}=1$.

We now show that if a continuous rule satisfies Property B over the entire domain $\mathcal{R}^{N}$ and the domain is connected, then the rule is diagonally dictatorial.

Lemma 2. If a continuous rule satisfies Property $B$ and the domain is connected, then it is diagonally dictatorial.

Proof. Let $\mathcal{R}^{N}$ be a connected domain. Assume that $\varphi: \mathcal{R}^{N} \rightarrow Z$ is continuous 
and satisfies Property B. Then by Lemma 1, for all diagonal profiles $R, \varphi(R)$ is extreme. Let $h: \mathcal{R} \rightarrow Z$ be defined as follows: for all $R_{0} \in \mathcal{R}, h\left(R_{0}\right) \equiv$ $\varphi\left(R_{0}, \cdots, R_{0}\right)$. By continuity of $\varphi, h$ is continuous. Since $\varphi$ chooses an extreme allocation at all diagonal profiles, $h$ has a finite range. Now we can apply the fact that if a continuous function defined over a connected space has a finite range in a Hausdorff space, then it is constant. Thus, $h$ is constant, that is, there exists an agent $i \in N$ such that for all $R_{0} \in \mathcal{R}, h_{i}\left(R_{0}\right)=\Omega$. Therefore agent $i$ is the diagonal dictator and $\varphi$ is diagonally dictatorial.

Remark 1. Suppose that $\mathcal{R}^{N}$ is not connected but is a union of connected subdomains. Then every continuous rule satisfying Property B over $\mathcal{R}^{N}$ is diagonally dictatorial over each of the subdomains. However the diagonal dictators may vary from subdomain to subdomain.

Adding veto-proofness, we obtain:

Lemma 3. If a continuous and veto-proof rule satisfies Property $B$ and the domain is connected, then it is dictatorial.

Proof. Let $\mathcal{R}^{N}$ be a connected domain. Assume that $\varphi: \mathcal{R}^{N} \rightarrow Z$ satisfies Property $\mathrm{B}$ and is continuous and veto-proof. By Lemma 2, $\varphi$ is diagonally dictatorial. Let $i^{*} \in N$ be the diagonal dictator. Without loss of generality, let $i^{*}=1$. We prove that for all $R \in \mathcal{R}^{N}, \varphi(R)=(\Omega, 0, \cdots, 0)$.

For all $R \in \mathcal{R}^{N}$, let $H(R) \equiv\left\{i \in N: R_{i} \neq R_{1}\right\}$ be the set of agents who have different preferences from agent 1's. Note that $H(R) \subseteq N \backslash\{1\}$ and $0 \leq|H(R)| \leq n-1$. In what follows, we prove the following statement, referred to as $S(k)$, by an induction argument with respect to $k \in\{0,1, \cdots, n-1\}$ : for all $R \in \mathcal{R}^{N}$, if $|H(R)| \leq k, \varphi(R)=(\Omega, 0, \cdots, 0)$.

The first step of the induction argument, $S(0)$, follows directly from Lemma 2. Now suppose $S(m)$ for $m \in\{1, \cdots, n-2\}$. In order to prove $S(m+1)$, we use the following claim.

Claim 1. For all $R \in \mathcal{R}^{N}$ with $|H(R)|=m+1$, if $i \in H(R)$, then $\varphi_{i}(R)=0$.

Proof. Let $R \in \mathcal{R}^{N}$ be given as above. Let $z \equiv \varphi(R)$. For each $i \in H(R)$, let $R_{i}^{\prime}=R_{1}$. Then $\left|H\left(R_{i}^{\prime}, R_{-i}\right)\right|=m$ and so by the induction hypothesis, $\varphi_{i}\left(R_{i}^{\prime}, R_{-i}\right)=0$. Hence for all $i \in H(R)$, if $z_{i} \neq 0$, then agent $i$ with preference $R_{i}^{\prime}$ can escape the worst outcome 0 by reporting $R_{i}$, contradicting veto-proofness.

To complete the final step, let $\bar{R} \in \mathcal{R}^{N}$ be such that $|H(\bar{R})|=m+1$. Let $j \in H(\bar{R})$ (since $m \geq 1$, there exists such $j$ ). For all $R_{j}^{\prime} \in \mathcal{R}$, let $g\left(R_{j}^{\prime}\right) \equiv$ 
$\varphi\left(R_{j}^{\prime}, \bar{R}_{-j}\right)$. If $R_{j}^{\prime}=\bar{R}_{1}$, then $\left|H\left(R_{j}^{\prime}, \bar{R}_{-j}\right)\right|=m$. Hence by the induction hypothesis, $g\left(R_{j}^{\prime}\right)=(\Omega, 0, \cdots, 0)$. If $R_{j}^{\prime} \neq \bar{R}_{1}$, then $\left|H\left(R_{j}^{\prime}, \bar{R}_{-j}\right)\right|=m+1$. Hence by Claim $1, \sum_{i \in N \backslash H\left(R_{j}^{\prime}, \bar{R}_{-j}\right)} \varphi_{i}\left(R_{j}^{\prime}, \bar{R}_{-j}\right)=\Omega$. Since all agents in $N \backslash H\left(R_{j}^{\prime}, \bar{R}_{-j}\right)$ have the same preferences, then, by Lemma $1, g\left(R_{j}^{\prime}\right)$ is an extreme allocation. Therefore $g$ has a finite range containing $(\Omega, 0, \cdots, 0)$. Since $g$ is continuous and $\mathcal{R}$ is connected, the range of $g$ is a singleton set $\{(\Omega, 0, \cdots, 0)\}$. Therefore $\varphi(\bar{R})=g\left(\bar{R}_{j}\right)=(\Omega, 0, \cdots, 0)$.

Remark 2. When the domain $\mathcal{R}^{N}$ is not connected, Lemma 3 does not hold. Let $\mathcal{R}_{a}^{*}, \mathcal{R}_{b}^{*} \subseteq \mathcal{R}$ constitute a disconnection of $\mathcal{R} \equiv \mathcal{R}_{a}^{*} \cup \mathcal{R}_{b}^{*}$. Let $N \equiv\{1,2,3\}$. Define a rule $\varphi$ as follows: for all $R \in \mathcal{D}$, (i) if $R_{3} \in \mathcal{R}_{a}^{*}, \varphi(R) \equiv(\Omega, 0,0)$ and (ii) if $R_{3} \in \mathcal{R}_{b}^{*}, \varphi(R) \equiv(0, \Omega, 0)$. Then $\varphi$ satisfies Property B over $\mathcal{R}^{N}$ and is continuous, and strategy-proof. However, $\varphi$ is not dictatorial.

Let $A$ be an arbitrary index set. A family of subsets of $\mathcal{R},\left\{\mathcal{R}_{\alpha} \subseteq \mathcal{R}: \alpha \in A\right\}$, is union-dense if $\cup_{\alpha \in A} \mathcal{R}_{\alpha}$ is dense in $\mathcal{R}$. We next show that if $\mathcal{R}$ has a uniondense family of connected subsets, $\left\{\mathcal{R}_{\alpha} \subseteq \mathcal{R}: \alpha \in A\right\}$, then every continuous rule that satisfies Property B over $\mathcal{R}_{\alpha}^{N}$ for all $\alpha \in A$ is diagonally dictatorial.

Lemma 4. Assume that $\mathcal{R}$ is connected and has a union-dense family of connected subsets, $\left\{\mathcal{R}_{\alpha} \subseteq \mathcal{R}: \alpha \in A\right\}$. Then every continuous rule satisfying Property $B$ over $\mathcal{R}_{\alpha}^{N}$ for all $\alpha \in A$, is diagonally dictatorial.

Proof. Let $\varphi: \mathcal{R}^{N} \rightarrow Z$ be continuous and satisfy Property B over $\mathcal{R}_{\alpha}^{N}$ for all $\alpha \in A$. Then by Lemma 2 , for all $\alpha \in A, \varphi$ is diagonally dictatorial over $\mathcal{R}_{\alpha}^{N}$. Since $\left\{\mathcal{R}_{\alpha}: \alpha \in A\right\}$ is union-dense, then for each $R_{0} \in \mathcal{R}$, there exists a sequence of preferences $\left(R_{0}^{n}: n \in \mathbb{N}\right)$ in $\cup_{\alpha \in A} \mathcal{R}_{\alpha}$, converging to $R_{0}$. Since for all $n \in \mathbb{N}$, $\varphi\left(R_{0}^{n}, \cdots, R_{0}^{n}\right)$ is extreme and $\varphi$ is continuous, then $\varphi\left(R_{0}, \cdots, R_{0}\right)$ is also extreme. Therefore $\varphi$ chooses an extreme allocation at all diagonal profiles in $\mathcal{R}^{N}$. For all $R_{0} \in \mathcal{R}$, let $h\left(R_{0}\right) \equiv \varphi\left(R_{0}, \cdots, R_{0}\right)$. Then the range of $h$ is a finite set of extreme allocations. Since $\varphi$ is continuous, $h$ is also continuous. Therefore since $\mathcal{R}$ is connected, the range of $h$ is a singleton. That is, there exists $i \in N$ such that for all $R_{0} \in \mathcal{R}, \varphi_{i}\left(R_{0}, \cdots, R_{0}\right)=\Omega$.

Adding veto-proofness, we obtain:

Lemma 5. Assume that $\mathcal{R}$ is connected and has a union-dense family of connected subsets, $\left\{\mathcal{R}_{\alpha} \subseteq \mathcal{R}: \alpha \in A\right\}$. Then every continuous and veto-proof rule that satisfies Property B over $\mathcal{R}_{\alpha}^{N}$ for all $\alpha \in A$ is dictatorial. 
Proof. Let $\varphi: \mathcal{D} \rightarrow Z$ satisfy Property B over $\mathcal{R}_{\alpha}^{N}$ for each $\alpha \in A$ and be continuous and veto-proof. By Lemma 3, for all $\alpha \in A, \varphi$ is dictatorial over $\mathcal{R}_{\alpha}^{N}$. Since $\cup_{\alpha \in A} \mathcal{R}_{\alpha}^{N}$ is dense in $\mathcal{R}$ and $\varphi$ is continuous, then the range of $\varphi$ is composed of finite extreme allocations. By continuity of $\varphi$ and connectedness of $\mathcal{R}$, we conclude that the range is a singleton: that is, $\varphi$ is dictatorial.

\section{The main results}

We show that every efficient and continuous rule over the linear domain satisfies Property B. Therefore, by Lemmas 2 and 3, we obtain the following results. Over the linear domain, (i) every efficient and continuous rule is diagonally dictatorial (Theorem 1) and (ii) a rule is efficient, continuous, and veto-proof if and only if it is dictatorial (Theorem 2). It follows from the first result that for any domain including the linear domain, there is no efficient and continuous rule satisfying any of the following equity criteria, equal treatment of equals, no-envy, and the equal division lower bound property (Corollary 1). Also it follows from the second result that a rule over the linear domain is efficient, continuous, and (weakly) strategy-proof if and only if it is dictatorial (Corollary 2). Adding "non-bossiness" (to be defined later) introduced by Satterthwaite and Sonnenschein (1981), we show that for any domain including the linear domain, a rule is efficient, continuous, veto-proof* (or weakly strategy-proof), and non-bossy if and only if it is dictatorial (Theorem 3). The homothetic domain contains the linear domain and so Corollary 1 and Theorem 3 apply. In the 2-good case, we show that non-bossiness in Theorem 3 is redundant and moreover, veto-proofness* (or weak strategy-proofness) can be weakened to veto-proofness. More precisely, we show that (i) every efficient and continuous rule over the homothetic domain is diagonally dictatorial (Theorem 4) and (ii) a rule is efficient, continuous, and veto-proof if and only if it is dictatorial (Theorem 5). Veto-proofness in the second result can be replaced with any one of the three requirements, veto-proofness ${ }^{*}$, weak strategy-proofness, and strategy-proofness.

We now show that every efficient and continuous rule over the linear domain is diagonally dictatorial.

Theorem 1. Every efficient and continuous rule over the linear domain is diagonally dictatorial.

Proof. Since $\mathcal{R}_{L}$ connected, then by Lemma 2, we only have to show that every efficient and continuous rule over $\mathcal{R}_{L}^{N}$ satisfies Property B. 
Let $\varphi: \mathcal{R}_{L}^{N} \rightarrow Z$ be efficient and continuous. We show that $\varphi$ satisfies parts (i)-(iii) in the definition of Property $\mathrm{B}$, with respect to $\left\{e_{1}, \cdots, e_{l}\right\}$. Parts (i) and (iii) are trivially satisfied. To show part (ii), let $R \in \mathcal{R}_{L}^{N}$ be such that for two agents $i$ and $j$ with $i \neq j, R_{i}=R_{j}$. Let $z \equiv \varphi(R)$. We only have to show that for all $k, m \in\{1, \cdots, l\}$ with $k \neq m$, either $z_{i k}=0$ or $z_{j m}=0$. Suppose by contradiction that there exist $k, m \in\{1, \cdots, l\}$ such that $k \neq m, z_{i k}>0$, and $z_{j m}>0$. Without loss of generality, we set $k=1$ and $m=2$.

Let $\hat{\Omega} \equiv z_{i}+z_{j}$. Then $\hat{\Omega}_{1}>0$ and $\hat{\Omega}_{2}>0$. Let $R_{0} \equiv R_{i}=R_{j}$. Let $p_{0} \in \mathbb{R}_{++}^{l}$ represent $R_{0}$. Let $d$ be a real number satisfying $0<d<\frac{1}{2} \hat{\Omega}_{1}$. Let $B(\hat{\Omega}, d) \equiv$ $\left\{z_{0}^{\prime} \in \mathbb{R}_{+}^{l}:\left\|z_{0}^{\prime}-\hat{\Omega}\right\| \leq d\right\}$ be the closed ball with center $\hat{\Omega}$ and radius $d$. Let $A \equiv\left\{z_{i}^{\prime} \in Z_{0}: z_{i 1}^{\prime} \in\left[\hat{\Omega}_{1}-d, \hat{\Omega}_{1}+d\right]\right.$ or $\left.z_{i 2}^{\prime}=0\right\}$ and $B \equiv\left\{z_{i}^{\prime} \in Z_{0}: z_{i 1}^{\prime}=0\right.$ or $\left.z_{i 2}^{\prime} \in\left[\hat{\Omega}_{2}-d, \hat{\Omega}_{2}+d\right]\right\}$. We show that both $z_{i}$ and $z_{j}$ are in $A \cap B$.

For all $\delta>0$, let $p_{0}^{\delta} \equiv\left(p_{01}+\delta, p_{02}, \cdots, p_{0 l}\right)$ and $R_{0}^{\delta}$ be the linear preference represented by $p_{0}^{\delta}$. Let $R^{\delta}$ be a profile such that $R_{i}^{\delta} \equiv R_{0}^{\delta}$ and for all $h \neq i$, $R_{h}^{\delta} \equiv R_{h}$. Let $z^{\delta} \equiv \varphi\left(R^{\delta}\right)$. Note that $\lim _{\delta \rightarrow 0} R^{\delta}=R$. Hence by continuity of $\varphi$, there exists $\bar{\delta}>0$ such that for all $\delta \in(0, \bar{\delta}), z_{i}^{\delta}+z_{j}^{\delta} \in B(\hat{\Omega}, d)$.

Claim 1. For all $\delta \in(0, \bar{\delta}), z_{i}^{\delta} \in A$.

Proof. Since $z_{i}^{\delta}, z_{j}^{\delta} \in \mathbb{R}_{+}^{l}$ and $z_{i}^{\delta}+z_{j}^{\delta} \in B(\hat{\Omega}, d), z_{i 1}^{\delta} \leq \hat{\Omega}_{1}+d$. Hence if we assume $z_{i}^{\delta} \notin A$, then $z_{i 1}^{\delta}<\hat{\Omega}_{1}-d$ and $z_{i 2}^{\delta}>0$. Since $z_{i}^{\delta}+z_{j}^{\delta} \in B(\hat{\Omega}, d), z_{j 1}^{\delta}>0$. Since $z_{i 2}^{\delta}>0, z_{j 2}^{\delta}<\Omega_{2}$.

In the following, we define a feasible allocation that Pareto dominates $z^{\delta}$. Let $z_{i}^{\prime} \equiv\left(z_{i 1}^{\delta}+\rho, z_{i 2}^{\delta}-\rho \cdot p_{01} / p_{02}, z_{i 3}^{\delta}, \cdots, z_{i l}^{\delta}\right)$ and $z_{j}^{\prime} \equiv\left(z_{j 1}^{\delta}-\rho, z_{j 2}^{\delta}+\rho \cdot p_{01} / p_{02}, z_{j 3}^{\delta}, \cdots, z_{j l}^{\delta}\right)$. For all $h \in N \backslash\{i, j\}$, let $z_{h}^{\prime}=z_{h}^{\delta}$. Note that $\sum_{N} z_{i}^{\prime}=\sum_{N} z_{i}^{\delta}=\Omega$. Since $z_{i 1}^{\delta} \leq \hat{\Omega}_{1}-d<\Omega_{1}, z_{i 2}^{\delta}>0, z_{j 1}^{\delta}>0$, and $z_{j 2}^{\delta}<\Omega_{2}$, there exists $\rho>0$ such that $z^{\prime}$ is feasible. By definition of $R^{\delta}$, it is easy to show that $z_{i}^{\prime} P_{i}^{\delta} z_{i}^{\delta}$ and for all $h \neq i, z_{h}^{\prime} I_{h}^{\delta} z_{h}^{\delta}$. Hence $z^{\prime}$ Pareto dominates $z^{\delta}$. This contradicts efficiency of $\varphi$.

By continuity of $\varphi, \lim _{\delta \rightarrow 0} z_{i}^{\delta}=z_{i}$. Since for all $\delta \in(0, \bar{\delta}), z_{i}^{\delta} \in A$ and $A$ is closed, $z_{i} \in A$.

For all $\delta>0$, let $\hat{p}_{0}^{\delta} \equiv\left(p_{01}-\delta, p_{02}, \cdots, p_{0 l}\right)$ and let $\hat{R}_{0}^{\delta}$ be the linear preference represented by $\hat{p}_{0}^{\delta}$. Let $\hat{R}^{\delta}$ be the profile defined by $\hat{R}_{i}^{\delta} \equiv \hat{R}_{0}^{\delta}$ and for all $h \neq i$, $\hat{R}_{h}^{\delta} \equiv \hat{R}_{h}$. Let $\hat{z}^{\delta} \equiv \varphi\left(\hat{R}^{\delta}\right)$. Note that $\lim _{\delta \rightarrow 0} \hat{R}^{\delta}=R$. Hence by continuity of $\varphi$, there exists $\hat{\delta}>0$ such that for all $\delta \in(0, \hat{\delta}), \hat{z}_{i}^{\delta}+\hat{z}_{j}^{\delta} \in B(\hat{\Omega}, d)$. Now using the same argument as in Claim 1 , we show that for all $\delta \in(0, \hat{\delta}), \hat{z}_{i}^{\delta} \in B$. Therefore by continuity of $\varphi$ and closedness of $B$, we have $z_{i} \in B$.

Therefore $z_{i} \in A \cap B$. In order to prove $z_{j} \in A \cap B$, we apply the same argument as above.

Since $z_{i} \in A \cap B$, either (i) $z_{i 1}=0$ and $z_{i 2}=0$, or (ii) $z_{i 1} \in\left[\hat{\Omega}_{1}-d, \hat{\Omega}_{1}+d\right]$ 
and $z_{i 2} \in\left[\hat{\Omega}_{2}-d, \hat{\Omega}_{2}+d\right]$. Since $z_{i 1}>0$ by the initial assumption, (i) does not hold. Therefore $z_{i 1} \in\left[\hat{\Omega}_{1}-d, \hat{\Omega}_{1}+d\right]$ and $z_{i 2} \in\left[\hat{\Omega}_{2}-d, \hat{\Omega}_{2}+d\right]$.

Similarly, we show that $z_{j 1} \in\left[\hat{\Omega}_{1}-d, \hat{\Omega}_{1}+d\right]$ and $z_{j 2} \in\left[\hat{\Omega}_{2}-d, \hat{\Omega}_{2}+d\right]$. Hence $z_{i 1}+z_{j 1} \geq 2 \hat{\Omega}_{1}-2 d$. Therefore since $d<\frac{1}{2} \hat{\Omega}_{1}$, then $z_{i 1}+z_{j 1} \geq 2 \hat{\Omega}_{1}-2 d>\hat{\Omega}_{1}=$ $z_{i 1}+z_{j 1}$. This is a contradiction.

If an allocation rule is diagonally dictatorial, it violates standard equity properties such as equal treatment of equals, no-envy, the equal division lower bound property, etc.

Corollary 1. Given any domain containing the linear domain, there exists no efficient and continuous rule satisfying any one of the three equity criteria, equal treatment of equals, no-envy, and the equal division lower bound property.

The impossibility is because of extreme choices for diagonal preference profiles. And the set of diagonal preference profiles is a "measure zero" subset of the linear domain. Thus, despite the impossibility, there may exist efficient and continuous rules that behave "nicely" over the set of non-diagonal profiles. ${ }^{11}$ Our next result is that if we require veto-proofness in addition, we cannot escape dictatorship. The proof is immediate from Lemma 3 and Proof of Theorem 1.

\footnotetext{
${ }^{11}$ For example, in the two agents and two goods economy, let $\varepsilon>0$ be an arbitrary real
} number and $\mathcal{D}_{\varepsilon} \equiv\left\{\left(R_{1}, R_{2}\right) \in \mathcal{R}_{L}^{2}:\left\|p_{1}-p_{2}\right\|>\varepsilon\right.$, where $p_{1} \in \Delta^{1}$ and $p_{2} \in \Delta^{1}$ represent $R_{1}$ and $R_{2}$ respectively\}. For each $p_{1}, p_{2} \in \Delta^{1}$ with $p_{1} \neq p_{2}$, let $z\left(p_{1}, p_{2}\right)$ be the efficient allocation that is indifferent for agent 1 to the equal division (thus when $p_{11}>p_{21}, z_{1}\left(p_{1}, p_{2}\right)$ is the intersection of the 1's indifference curve through $\Omega / 2$ and the "lower right corner" of the Edgeworth box; when $p_{11}<p_{21}, z_{1}\left(p_{1}, p_{2}\right)$ is on the "upper left corner" of the Edgeworth box). For each $\delta \in[0, \varepsilon]$, let $z_{1}^{l r}\left(p_{1}, \delta\right)$ be a bundle on the lower right corner of the Edgeworth box such that $z_{1}^{l r}\left(p_{1}, \delta\right)$ moves continuously from $z_{1}\left(p_{1}, p_{2}\right)$ to $\Omega$ as $\delta$ changes from $\varepsilon$ to 0 , for some $p_{2}$ with $p_{21}<p_{11}$. Thus, $z_{1}^{l r}\left(p_{1}, 0\right)=\Omega$ and $z_{1}^{l r}\left(p_{1}, \varepsilon\right)=z_{1}\left(p_{1}, p_{2}\right)$. Similarly, for each $\delta \in[0, \varepsilon]$, let $z_{1}^{u l}\left(p_{1}, \delta\right)$ be a bundle on the upper left corner such that $z_{1}^{u l}\left(p_{1}, \delta\right)$ moves continuously from $z_{1}\left(p_{1}, p_{2}\right)$ to $\Omega$ as $\delta$ changes from $\varepsilon$ to 0 , for some $p_{2}$ with $p_{21}>p_{11}$.

Now define $\varphi$ as follows. For all $\left(R_{1}, R_{2}\right) \in \mathcal{D}_{\varepsilon}, \varphi_{1}\left(R_{1}, R_{2}\right) \equiv z_{1}\left(p_{1}, p_{2}\right)$, where $p_{1}, p_{2} \in \Delta^{1}$ represent $R_{1}$ and $R_{2}$ respectively. When $\left\|p_{1}-p_{2}\right\| \leq \varepsilon$,

$$
\varphi_{1}\left(R_{1}, R_{2}\right) \equiv\left\{\begin{array}{l}
z_{1}^{l r}\left(p_{1},\left\|p_{1}-p_{2}\right\|\right), \text { if } p_{11} \geq p_{21} \\
z_{1}^{u l}\left(p_{1},\left\|p_{1}-p_{2}\right\|\right), \text { if } p_{11}<p_{21}
\end{array}\right.
$$

For each $\left(R_{1}, R_{2}\right)$, let $\varphi_{2}\left(R_{1}, R_{2}\right) \equiv \Omega-\varphi_{1}\left(R_{1}, R_{2}\right)$. By definition, $\varphi$ satisfies efficiency and continuity. Clearly, over $\mathcal{D}_{\varepsilon}, \varphi$ satisfies the equal division lower bound property. However, $\varphi$ is diagonally dictatorial, since it gives $\Omega$ to agent 1 at each diagonal profile. Thus, although $\varphi$ is diagonally dictatorial, $\varphi$ violates the equal division lower bound property only over $\mathcal{R}_{L}^{2} \backslash \mathcal{D}_{\varepsilon}$, which can be made arbitrarily small by choosing sufficiently small $\varepsilon>0$. 
Theorem 2. A rule over the linear domain is efficient, continuous, and vetoproof if and only if it is dictatorial.

Since veto-proofness* [or (weak) strategy-proofness] implies veto-proofness, we have the following corollary:

Corollary 2. (i) A rule over the linear domain is efficient, continuous, and veto-proof* if and only if it is dictatorial. (ii) A rule over the linear domain is efficient, continuous, and (weakly) strategy-proof if and only if it is dictatorial.

Examples 1-3 below establish independence of the three requirements in each of Theorem 2 and Corollary 2. For simplicity, we assume $N=\{1,2,3\}$ and $l=2$. Then we may represent each linear preference $R_{i}$ by a number $p_{i} \in \mathbb{R}_{++}$, where $\left(p_{i}, 1\right)$ is the normal vector of indifference curves of $R_{i}$. In the following examples, we use $p_{i}$ instead of $R_{i}$.

Example 1. Define $\varphi$ as follows: (i) if $p_{3} \geq 1, \varphi\left(p_{1}, p_{2}, p_{3}\right) \equiv(\Omega, 0,0)$, (ii) if $p_{3}<1, \varphi\left(p_{1}, p_{2}, p_{3}\right) \equiv(0, \Omega, 0)$. Then $\varphi$ satisfies efficiency and strategy-proofness.

Example 2. Define $\varphi$ as follows: (i) if $p_{1}>\max \left\{p_{2}, p_{3}\right\}, \varphi_{1}\left(p_{1}, p_{2}, p_{3}\right) \equiv\left(\Omega_{1}, 0\right)+$ $\frac{\max \left\{p_{2}, p_{3}\right\}}{p_{1}} \cdot\left(0, \Omega_{2}\right)$ and for all $i=2,3, \varphi_{i}\left(p_{1}, p_{2}, p_{3}\right) \equiv \frac{1}{2}\left(1-\frac{\max \left\{p_{2}, p_{3}\right\}}{p_{1}}\right) \cdot\left(0, \Omega_{2}\right)$, (ii) if $p_{1}<\min \left\{p_{2}, p_{3}\right\}, \varphi_{1}\left(p_{1}, p_{2}, p_{3}\right) \equiv \frac{p_{1}}{\min \left\{p_{2}, p_{3}\right\}} \cdot\left(\Omega_{1}, 0\right)+\left(0, \Omega_{2}\right)$ and for all $i=2,3, \varphi_{i}\left(p_{1}, p_{2}, p_{3}\right) \equiv \frac{1}{2}\left(1-\frac{p_{1}}{\min \left\{p_{2}, p_{3}\right\}}\right) \cdot\left(\Omega_{1}, 0\right)$, and (iii) in all other cases, $\varphi\left(p_{1}, p_{2}, p_{3}\right) \equiv(\Omega, 0,0)$. It is easy to show that $\varphi$ satisfies efficiency and continuity.

Example 3. Constant allocation rules satisfy continuity and strategy-proofness trivially.

Theorem 2 and Corollary 2 pertain to the linear domain. However, for any larger domain, these two results are still applicable at least locally over the linear domain. Considering the following additional requirement introduced by Satterthwaite and Sonnenschein (1981), we extend the two results to any domain including the linear domain. A rule $\varphi: \mathcal{R}^{N} \rightarrow Z$ is non-bossy if no one can affect others' bundles without affecting his own bundle, that is, for all $R \in \mathcal{R}^{N}$ and all $i \in N$, there is no $R_{i}^{\prime} \in \mathcal{R}$ such that $\varphi_{i}\left(R_{i}^{\prime}, R_{-i}\right)=\varphi_{i}(R)$ and $\varphi_{-i}\left(R_{i}^{\prime}, R_{-i}\right) \neq \varphi_{-i}(R)$.

Theorem 3. Given any domain including the linear domain, (i) a rule is efficient, continuous, veto-proof*, and non-bossy if and only if it is dictatorial; (ii) a rule is efficient, continuous, weakly strategy-proof, and non-bossy if and only if it is dictatorial. 
Proof. We only have to show (i). Let $\mathcal{R} \supseteq \mathcal{R}_{L}^{N}$ be a family of preferences. Let $\varphi: \mathcal{R}^{N} \rightarrow Z$ be efficient, continuous, veto-proof*, and non-bossy. Then by Theorem 2 , there exists $i \in N$ such that for all $R \in \mathcal{R}_{L}^{N}, \varphi_{i}(R)=\Omega$. Let $R \in \mathcal{R}^{N}$. We only have to show $\varphi_{i}(R)=\Omega$.

Let $\bar{R} \in \mathcal{R}_{L}^{N}$. Then $\varphi_{i}(\bar{R})=\Omega$. Let $j \in N \backslash i$. Since $\varphi_{j}(\bar{R})=0$, then by veto-

proofness*, $\varphi_{j}\left(R_{j}, \bar{R}_{-j}\right)=0$. By non-bossiness, $\varphi\left(R_{j}, \bar{R}_{-j}\right)=\varphi(\bar{R})$. Applying the same argument successively to each agent in $N \backslash i$, we show $\varphi\left(\bar{R}_{i}, R_{-i}\right)=$ $\varphi(\bar{R})$. Then $\varphi_{i}\left(\bar{R}_{i}, R_{-i}\right)=\Omega$. Applying veto-proofness ${ }^{*}$ to agent $i, \varphi_{i}\left(R_{i}, R_{-i}\right)=$ $\Omega$.

Remark 3. (i) In the 2-agent case, non-bossiness is redundant. Given any domain including the linear domain, a rule is efficient, continuous, and veto-proof* [or, weakly strategy-proof] if and only if it is dictatorial.

(ii) When veto-proofness* or weak strategy-proofness is replaced with strategyproofness, similar result holds without continuity. Serizawa (2000b) shows that a rule is efficient, strategy-proof, and non-bossy if and only if it is dictatorial.

The homothetic domain contains the linear domain and so Corollary 1 and Theorem 3 apply. Moreover, in the 2-good case, we establish stronger results.

We consider the following union-dense family of connected subsets of the homothetic domain. We will show later that every efficient and continuous rule satisfies Property B over each of these subsets.

We first introduce useful notation. Let $\varepsilon>0$. If $\Omega-(\varepsilon, 0) \in \mathbb{R}_{+}^{2}$, then let $a_{1}^{\varepsilon} \equiv \Omega-(\varepsilon, 0)$. Otherwise, let $a_{1}^{\varepsilon} \equiv\left(0, \Omega_{2}\right)$. If $\Omega-(0, \varepsilon) \in \mathbb{R}_{+}^{2}$, then let $a_{2}^{\varepsilon} \equiv \Omega-(0, \varepsilon)$. Otherwise, let $a_{2}^{\varepsilon} \equiv\left(\Omega_{1}, 0\right)$.

The two vectors, $a_{1}^{\varepsilon}$ and $a_{2}^{\varepsilon}$, generate a positive cone $C^{\varepsilon} \equiv\left\{\alpha a_{1}^{\varepsilon}+\beta a_{2}^{\varepsilon}: \alpha, \beta \in\right.$ $\left.\mathbb{R}_{+}\right\}$.

Let $X \subseteq \mathbb{R}_{+}^{2}$ and $R_{0}, R_{0}^{\prime} \in \mathcal{R}_{H}$. We say that two preferences, $R_{0}$ and $R_{0}^{\prime}$, coincide on $X$ if the two preferences order every two bundles in $X$ in the same way. Let $x \in X$. A vector $p \in \mathbb{R}_{+}^{2}$ is a supporting normal vector at $x$ for $R_{0}$ if $p$ is normal to the hyperplane through $x$, which supports the upper contour set (at $x$ ) of $R_{0}$, formally, for all $y \in \mathbb{R}_{+}^{2}$ with $y R_{0} x, p \cdot y \geq p \cdot x$. We say that two preferences $R_{0}$ and $R_{0}^{\prime}$ have identical supporting normal vectors on $X$ if for all $x \in X$, the set of supporting normal vectors at $x$ for $R_{0}$ is equal to the set of supporting normal vectors at $x$ for $R_{0}^{\prime}$. We say that $R_{0}$ is strictly convex over $X$ if for all $x, x^{\prime} \in X$ and all $\lambda \in(0,1)$, if $\lambda x+(1-\lambda) x^{\prime} \in X$ and $x^{\prime} R_{0} x$, then $\left(\lambda x+(1-\lambda) x^{\prime}\right) P_{0} x$.

Next we define a class of homothetic preferences that are locally linear over the cone and strictly convex outside the cone. Formally, let $\mathcal{R}_{H, \varepsilon} \subseteq \mathcal{R}_{H}$ be the 
class of preferences defined as follows: for all $R_{0} \in \mathcal{R}_{H}, R_{0} \in \mathcal{R}_{H, \varepsilon}$ if and only if (i) $R_{0}$ is strictly convex on $\mathbb{R}_{+}^{2} \backslash C^{\varepsilon}$, (ii) there exists a linear preference $R_{0}^{\prime} \in \mathcal{R}_{L}$ such that $R_{0}$ coincide with $R_{0}^{\prime}$ on $C^{\varepsilon}$, and (iii) the indifference curves of $R_{0}$ have kinks along both of the two rays, $\overrightarrow{0, a_{1}^{\varepsilon}}$ and $\overrightarrow{0, a_{2}^{\varepsilon}}$.

Let $\mathcal{R}_{H, s c}$ be the class of all strictly convex preferences in $\mathcal{R}_{H}$. Note that for all $R_{0} \in \mathcal{R}_{H, s c}$, there exists a unique preference in $\mathcal{R}_{H, \varepsilon}$ that coincides with $R_{0}$ on $\mathbb{R}_{+}^{2} \backslash C^{\varepsilon}$. However for each $R_{0} \in \mathcal{R}_{H, \varepsilon}$, there are many preferences in $\mathcal{R}_{H, s c}$ that coincide with $R_{0}$ on $\mathbb{R}_{+}^{2} \backslash C^{\varepsilon}$. Hence we may say that $\mathcal{R}_{H, \varepsilon}$ is smaller than $\mathcal{R}_{H, s c}$.

We first show that if an efficient allocation has $i$-th component that lies outside $C^{\varepsilon}$ and two preferences in $\mathcal{R}_{H, \varepsilon}$ of agents $i$ and $j$ have identical supporting normal vectors outside $C^{\varepsilon}$, then their bundles are along the same ray through the origin. $^{12}$

Lemma 6. Let $z$ be efficient for $R \in \mathcal{R}_{H, \varepsilon}^{N}$. For all $i, j \in N$, if $R_{i}$ and $R_{j}$ have identical supporting normal vectors over $\mathbb{R}_{+}^{2} \backslash C^{\varepsilon}$ and $z_{i} \notin C^{\varepsilon}$, then there exists $\alpha \in \mathbb{R}_{+}$such that $z_{j}=\alpha z_{i}$.

Proof. Let $z \in Z$ be efficient for $R \in \mathcal{R}_{H, \varepsilon}^{N}$. Let $i, j \in N$. Assume that $R_{i}$ and $R_{j}$ have identical supporting normal vectors over $\mathbb{R}_{+}^{2} \backslash C^{\varepsilon}$ and $z_{i} \notin C^{\varepsilon}$.

Claim 1. For all $x \in \mathbb{R}_{+}^{l}$ such that $x \neq z_{i}$ and $x I_{i} z_{i}$, there exists no vector that is a supporting normal vector for $R_{i}$ both at $x$ and at $z_{i}$.

Proof. Let $x I_{i} z_{i}$. Suppose to the contrary that there exists $p \in \mathbb{R}_{+}^{l}$ such that (i) for all $y \in \mathbb{R}_{+}^{l}$ with $y R_{i} z_{i}, p \cdot y \geq p \cdot z_{i}$ and (ii) for all $y \in \mathbb{R}_{+}^{l}$ with $y R_{i} x$, $p \cdot y \geq p \cdot x$. Then since $z_{i} I_{i} x, p \cdot z_{i}=p \cdot x$. Since $R_{i}$ is convex, for all $\delta \in(0,1)$, $\left[\delta x+(1-\delta) z_{i}\right] I_{i} z_{i}$. Since $C^{\varepsilon}$ is closed, there exists $\delta^{*} \in(0,1)$ such that for all $\delta \in$ $\left[0, \delta^{*}\right], \delta x+(1-\delta) z_{i} \notin C^{\varepsilon}$. Therefore for all $\lambda \in(0,1), \lambda\left(\delta^{*} x+\left(1-\delta^{*}\right) z_{i}\right)+(1-\lambda) z_{i} \notin$ $C^{\varepsilon}, \delta^{*} x+\left(1-\delta^{*}\right) z_{i} \notin C^{\varepsilon}$, and $\left[\lambda\left(\delta^{*} x+\left(1-\delta^{*}\right) z_{i}\right)+(1-\lambda) z_{i}\right] I_{i}\left[\delta^{*} x+\left(1-\delta^{*}\right) z_{i}\right]$ $I_{i} z_{i}$. This contradicts that $R_{i} \in \mathcal{R}_{H, \varepsilon}$ is strictly convex over $\mathbb{R}_{+}^{2} \backslash C^{\varepsilon}$.

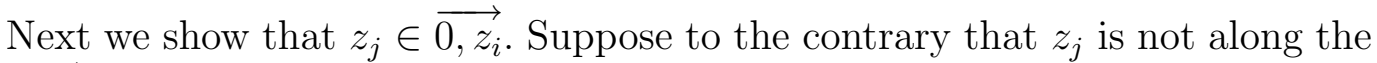
ray $\overrightarrow{0, z_{i}}$. Then, there exist $\alpha, \beta \in \mathbb{R}_{++}$such that $z_{i} I_{i} \alpha z_{j}$ and $z_{j} I_{j} \beta z_{i}$. Since $z_{j} \notin \overrightarrow{0, z_{i}}, z_{i} \neq \alpha z_{j}$ and $z_{j} \neq \beta z_{i}$. Since $z$ is efficient for $R$, there is $p \in \mathbb{R}_{+}^{l}$ that supports $R_{i}$ at $z_{i}$ and $R_{j}$ at $z_{j}$. Then by homotheticity, $p$ supports $R_{i}$ both at $z_{i}$ and at $\alpha z_{j}$, contradicting Claim 1 .

In the 2-good case, the following three simple facts hold: (i) $\mathcal{R}_{H}$ is pathconnected, (ii) for all $\varepsilon>0, \mathcal{R}_{H, \varepsilon}$ is path-connected, and (iii) $\cup_{\varepsilon>0} \mathcal{R}_{H}^{\varepsilon}$ is dense

\footnotetext{
${ }^{12}$ Schummer (1997) uses a similar fact for strictly convex and homothetic preferences.
} 
in $\mathcal{R}_{H}$. We sketch the proofs in Appendix. Using the three facts and applying Lemma 4 , we show that every efficient and continuous rule over $\mathcal{R}_{H}^{N}$ is diagonally dictatorial.

Theorem 4. In the 2-good case, every efficient and continuous rule over the homothetic domain is diagonally dictatorial.

Proof. By Lemma 4, we only have to show that every efficient and continuous rule over the homothetic domain satisfies Property B over $\mathcal{R}_{H, \varepsilon}^{N}$, for all $\varepsilon>0$.

Let $\varphi: \mathcal{R}_{H}^{N} \rightarrow Z$ be efficient and continuous. We show that $\varphi$ satisfies conditions (i)-(iii) of Property B over $\mathcal{R}_{H, \varepsilon}^{N}$, associated with the two linearly independent vectors, $a_{1}^{\varepsilon}$ and $a_{2}^{\varepsilon}$. Clearly, $\Omega \in \operatorname{int}\left\langle a_{1}^{\varepsilon}, a_{2}^{\varepsilon}\right\rangle_{+}$. Let $R \in \mathcal{R}_{H, \varepsilon}^{N}$ and $z \equiv \varphi(R)$. Assume that there exist $i, j \in N$ such that $i \neq j$ and $R_{i}=R_{j}$. Let $R_{0} \equiv R_{i}=R_{j}$. Let $p_{0} \in \mathbb{R}_{++}$and $R_{0}$ coincide with the linear preference represented by $\left(p_{0}, 1\right)$ $\in \mathbb{R}_{++}^{2}$ on $C^{\varepsilon}$. We only have to show that $z_{i}=0$ or $z_{j}=0$ or $z_{i}+z_{j} \notin$ int $\left\langle a_{1}^{\varepsilon}, a_{2}^{\varepsilon}\right\rangle_{+}$.

The proof is by contradiction. Suppose to the contrary that $z_{i} \neq 0, z_{j} \neq 0$, and $z_{i}+z_{j} \in \operatorname{int}\left\langle a_{1}^{\varepsilon}, a_{2}^{\varepsilon}\right\rangle_{+}\left(=\operatorname{int}\left(C^{\varepsilon}\right)\right)$. Let $z_{i}+z_{j} \equiv \hat{\Omega}$. Since $\hat{\Omega} \in \operatorname{int}\left(C^{\varepsilon}\right)$, there exists $\bar{\rho}$ such that for all $\rho \in(0, \bar{\rho})$, the closed ball $B(\hat{\Omega}, \rho)$ is contained in $C^{\varepsilon}$.

Our proof makes use of the following geometric objects. For all $B \subseteq \mathbb{R}^{2}$, let $\operatorname{comp}(B) \equiv\left\{x \in \mathbb{R}^{l}:\right.$ for some $\left.y \in B, x \leqq y\right\}$. Let $\rho \in(0, \bar{\rho})$ and $Z_{0}^{\rho} \equiv$ $Z_{0} \cap \operatorname{comp}(B(\hat{\Omega}, \rho))$. Let $H_{1}$ be the ray $\overrightarrow{0, a_{2}^{\varepsilon}}$ and $d_{1} \in \mathbb{R}^{2}$ be a vector such that $d_{1} \cdot a_{2}^{\varepsilon}=0$ and for all $z_{0} \in C^{\varepsilon}, d_{1} \cdot z_{0} \geq 0$. Let $H_{2}$ be the ray $\overrightarrow{0, a_{1}^{\varepsilon}}$ and $d_{2} \in \mathbb{R}^{2}$ be a vector such that $d_{2} \cdot a_{1}^{\varepsilon}=0$ and for all $z_{0} \in C^{\varepsilon}, d_{2} \cdot z_{0} \geq 0$. Note that since $a_{1}^{\varepsilon}$ and $a_{2}^{\varepsilon}$ are linearly independent, $d_{1}$ and $d_{2}$ are linearly independent also and that for all $z_{0} \in \mathbb{R}_{+}^{2}$, there exist $\alpha, \beta \in \mathbb{R}_{+}$such that $z_{0}=\alpha d_{1}+\beta d_{2}$.

Clearly, there exists a unique bundle $b_{1}^{\rho} \in B(\hat{\Omega}, \rho)$ such that for all $z_{0} \in$ $B(\hat{\Omega}, \rho), d_{1} \cdot z_{0} \geq d_{1} \cdot b_{1}^{\rho}$. Also, there exists a unique bundle $b_{2}^{\rho} \in B(\hat{\Omega}, \rho)$ such that for all $z_{0} \in B(\hat{\Omega}, \rho), d_{2} \cdot z_{0} \geq d_{2} \cdot b_{2}^{\rho} \cdot{ }^{13}$ Let $H_{1}^{\rho} \equiv\left\{z_{0} \in Z_{0}^{\rho}: d_{1} \cdot z_{0} \geq d_{1} \cdot b_{1}^{\rho}\right\}$ be the intersection of $Z_{0}^{\rho}$ and the half space above the line through $b_{1}^{\rho}$, which is normal to $d_{1}$. Let $H_{2}^{\rho} \equiv\left\{z_{0} \in Z_{0}^{\rho}: d_{2} \cdot z_{0} \geq d_{2} \cdot b_{2}^{\rho}\right\}$ be the intersection of $Z_{0}^{\rho}$ and the half space below the line through $b_{2}^{\rho}$, which is normal to $d_{2}$.

\footnotetext{
${ }^{13}$ Since $B(\hat{\Omega}, \rho)$ is compact and strictly convex, the following (a) and (b) have unique solutions.
}

$$
\begin{aligned}
& \text { (a) } \max _{z_{0} \in B(\hat{\Omega}, \rho)} d_{1} \cdot z_{0} \text {, } \\
& \text { (b) } \min _{z_{0} \in B(\hat{\Omega}, \rho)} d_{2} \cdot z_{0} \text {. }
\end{aligned}
$$

The solution for (a) is $b_{1}^{\rho}$ and the solution for (b) is $b_{2}^{\rho}$. 
In the following argument, we first prove that for sufficiently small $\rho>0$, we have $z_{i} \in\left(\overrightarrow{0, a_{1}^{\varepsilon}} \cup H_{1}^{\rho}\right) \cap\left(\overrightarrow{0, a_{2}^{\varepsilon}} \cup H_{2}^{\rho}\right)$. We next derive a contradiction based on this fact.

Since $R_{0}$ has kinks along both two rays, $\overrightarrow{0, a_{1}^{\varepsilon}}$ and $\overrightarrow{0, a_{2}^{\varepsilon}}$, then for some $\delta^{o}>0$, if $\delta \in\left(0, \delta^{o}\right)$, there exists $R_{0}^{\delta} \in \mathcal{R}_{H, \varepsilon}$ such that $R_{0}^{\delta}$ and $R_{0}$ have identical supporting normal vectors over $\mathbb{R}_{+}^{2} \backslash C^{\varepsilon},{ }^{14}$ and $R_{0}^{\delta}$ coincides with the linear preference represented by $\left(p_{0}-\delta, 1\right)$ over $C^{\varepsilon}$. Let $\delta \in\left(0, \delta^{o}\right)$. Let $R_{i}^{\delta} \equiv R_{0}^{\delta}$ and for all $h \neq i$, let $R_{h}^{\delta} \equiv R_{h}$. Let $z^{\delta} \equiv \varphi\left(R^{\delta}\right)$.

By continuity of $\varphi$, there exists $\bar{\delta} \in\left(0, \delta^{o}\right)$ such that for all $\delta \in(0, \bar{\delta}), z_{i}^{\delta}+z_{j}^{\delta} \in$ $B(\hat{\Omega}, \rho)$.

Claim 1. For all $\delta \in(0, \bar{\delta})$, we have $z_{i}^{\delta}, z_{j}^{\delta} \in C^{\varepsilon} \cap Z_{0}^{\rho}$.

Proof. Since $z_{i}^{\delta}+z_{j}^{\delta} \in B(\hat{\Omega}, \rho)$, clearly we have $z_{i}^{\delta}, z_{j}^{\delta} \in Z_{0}^{\rho}$. We only have to show that $z_{i}^{\delta}, z_{j}^{\delta} \in C^{\varepsilon}$. Since $B(\hat{\Omega}, \rho) \subseteq C^{\varepsilon}$, we have $z_{i}^{\delta}+z_{j}^{\delta} \in C^{\varepsilon}$. By definition, $R_{i}^{\delta}$ and $R_{j}^{\delta} \equiv R_{0}$ have identical supporting normal vectors on $\mathbb{R}_{+}^{2} \backslash C^{\varepsilon}$. Since $\varphi$ is efficient, $z^{\delta}$ is an efficient allocation. Therefore if $z_{i}^{\delta} \notin C^{\varepsilon}$, then by Lemma 6 , there exists $\alpha \geq 0, z_{j}^{\delta}=\alpha z_{i}^{\delta}$. Then $z_{i}^{\delta}+z_{j}^{\delta} \notin C^{\varepsilon}$, contradicting $z_{i}^{\delta}+z_{j}^{\delta} \in B(\hat{\Omega}, \rho) \subseteq$ $C^{\varepsilon}$. Therefore $z_{i}^{\delta} \in C^{\varepsilon}$.

Using the same argument, we show that $z_{j}^{\delta} \in C^{\varepsilon}$.

Claim 2. For all $\delta \in(0, \bar{\delta})$, we have $z_{i}^{\delta} \in \overrightarrow{0, a_{1}^{\varepsilon}} \cup H_{1}^{\rho}$.

Proof. Assume $z_{j}^{\delta} \neq 0$. If $z_{j}^{\delta} \in \overrightarrow{0, a_{1}^{\varepsilon}}$, then by efficiency, $z_{i}^{\delta} \in \overrightarrow{0, a_{1}^{\varepsilon}}$. So $z_{i}^{\delta}+z_{j}^{\delta} \in$ $\overrightarrow{0, a_{1}^{\varepsilon}}$, contradicting $z_{i}^{\delta}+z_{j}^{\delta} \in B(\hat{\Omega}, \rho)$. Hence if $z_{j}^{\delta} \neq 0$, then $z_{j}^{\delta} \notin \overrightarrow{0, a_{1}^{\varepsilon}}$. Therefore we distinguish three cases below.

Case 1. $z_{j}^{\delta}=0$. In this case, $z_{i}^{\delta} \in B(\hat{\Omega}, \rho)$. Hence by definition of $b_{1}^{\rho}, d_{1} \cdot z_{i}^{\delta} \geq$ $d_{1} \cdot b_{1}^{\rho}$. Therefore, $z_{i}^{\rho} \in H_{1}^{\rho}$.

Case 2. $z_{j}^{\delta} \neq 0$ and $z_{j}^{\delta} \in \operatorname{int}\left(C^{\varepsilon}\right) \cap Z_{0}^{\rho}$. In this case, by efficiency, $z_{i}^{\delta} \in \overrightarrow{0, a_{1}^{\varepsilon}}$.

Case 3. $z_{j}^{\delta} \neq 0$ and $z_{j}^{\delta} \in \overrightarrow{0, a_{2}^{\varepsilon}} \cap Z_{0}^{\rho}$. In this case, there exists $\alpha>0$ such that $z_{j}^{\delta}=\alpha a_{2}^{\varepsilon}$. Let $\Omega^{\delta} \equiv z_{i}^{\delta}+z_{j}^{\delta}$. Then $\Omega^{\delta} \in B(\hat{\Omega}, \rho)$ and $z_{i}^{\delta}=\Omega^{\delta}-\alpha a_{2}^{\varepsilon}$. Hence by definition of $b_{1}^{\rho}, d_{1} \cdot \Omega^{\delta} \geq d_{1} \cdot b_{1}^{\rho}$. Therefore $d_{1} \cdot z_{i}^{\delta}=d_{1} \cdot \Omega^{\delta}-\alpha\left(d_{1} \cdot a_{2}^{\varepsilon}\right)=d_{1} \cdot \Omega^{\delta} \geq d_{1} \cdot b_{1}^{\rho}$. Hence $z_{i}^{\delta} \in H_{1}^{\rho}$.

Therefore in all three cases, $z_{i}^{\delta} \in \overrightarrow{0, a_{1}^{\varepsilon}} \cup H_{1}^{\rho}$.

By continuity of $\varphi, \lim _{\delta \rightarrow 0} z_{i}^{\delta}=z_{i}$. Therefore since $\overrightarrow{0, a_{1}^{\varepsilon}} \cup H_{1}^{\rho}$ is closed, $z_{i} \in \overrightarrow{0, a_{1}^{\varepsilon}} \cup H_{1}^{\rho}$

\footnotetext{
${ }^{14}$ Even if $R_{0}^{\delta}$ and $R_{0}$ have identical supporting normal vectors over $\mathbb{R}_{+}^{2} \backslash C^{\varepsilon}, R_{0}^{\delta}$ is not necessarily equal to $R_{0}$ over $\mathbb{R}_{+}^{2} \backslash C^{\varepsilon}$.
} 
Since $R_{0}$ has kinks along both two rays, $\overrightarrow{0, a_{1}^{\varepsilon}}$ and $\overrightarrow{0, a_{2}^{\varepsilon}}$, then for some $\delta^{*}>$ 0 , if $\delta \in\left(0, \delta^{*}\right)$, there exists $\hat{R}_{0}^{\delta} \in \mathcal{R}_{H, \varepsilon}$ such that $\hat{R}_{0}^{\delta}$ and $R_{0}$ have identical supporting normal vectors over $\mathbb{R}_{+}^{2} \backslash C^{\varepsilon}$ and $\hat{R}_{0}^{\delta}$ coincides with the linear preference represented by $\left(p_{0}+\delta, 1\right)$ on $C^{\varepsilon}$. Let $\delta \in\left(0, \delta^{*}\right)$. Let $\hat{R}_{i}^{\delta}=\hat{R}_{0}^{\delta}$ and for all $h \neq i$, let $\hat{R}_{h}^{\delta}=R_{h}$. Let $\hat{z}^{\delta}=\varphi\left(\hat{R}^{\delta}\right)$. Now using $\hat{R}^{\delta}$ and $\hat{z}^{\delta}$ and following the same arguments as above, we prove that $z_{i} \in \overrightarrow{0, a_{2}^{\varepsilon}} \cup H_{2}^{\rho}$.

Therefore $z_{i} \in\left(\overrightarrow{0, a_{1}^{\varepsilon}} \cup H_{1}^{\rho}\right) \cap\left(\overrightarrow{0, a_{2}^{\varepsilon}} \cup H_{2}^{\rho}\right)$. Since for all $k, k^{\prime} \in\{1,2\}$ with $k \neq k^{\prime}, \overrightarrow{0, a_{k}^{\varepsilon}} \cap H_{k^{\prime}}^{\rho}=\varnothing$ and $\overrightarrow{0, a_{k}^{\varepsilon}} \cap \overrightarrow{0, a_{k^{\prime}}^{\varepsilon}}=\{0\}$, then $z_{i} \in\{0\} \cup\left[H_{1}^{\rho} \cap H_{2}^{\rho}\right]$. By assumption, $z_{i} \neq 0$. Therefore $z_{i} \in H_{1}^{\rho} \cap H_{2}^{\rho}$, that is, $d_{1} \cdot z_{i} \geq d_{1} \cdot b_{1}^{\rho}$ and $d_{2} \cdot z_{i} \geq d_{2} \cdot b_{2}^{\rho}$. Note that $\lim _{\rho \rightarrow 0} b_{1}^{\rho}=\lim _{\rho \rightarrow 0} b_{2}^{\rho}=\hat{\Omega}$. Taking limits in both sides of the two inequalities, $d_{1} \cdot z_{i} \geq d_{1} \cdot \hat{\Omega}$ and $d_{2} \cdot z_{i} \geq d_{2} \cdot \hat{\Omega}$. Since $\hat{\Omega}=z_{i}+z_{j}$, $d_{1} \cdot z_{j} \leq 0$ and $d_{2} \cdot z_{j} \leq 0$. Let $\alpha, \beta \in \mathbb{R}_{+}$be such that $z_{j}=\alpha d_{1}+\beta d_{2}$. Then from the two inequalities, we obtain $z_{j} \cdot z_{j} \leq 0$. This implies $z_{j}=0$, contradicting our assumption.

Using Lemma 5 and the same proof as in Theorem 4, we show that if we require veto-proofness in addition, then we cannot escape dictatorship.

Theorem 5. In the 2-good case, a rule over the homothetic domain is efficient, continuous, and veto-proof if and only if it is dictatorial.

Since each of veto-proofness ${ }^{*}$ and (weak) strategy-proofness implies veto-proofness, we have:

Corollary 3. In the 2-good case, (i) a rule over the homothetic domain is efficient, continuous, and veto-proof* if and only if it is dictatorial; (ii) a rule over the homothetic domain is efficient, continuous, and (weakly) strategy-proof if and only if it is dictatorial.

The independence of the requirements in each of Theorem 5 and Corollary 3 can be established easily.

\section{Discussion}

1. Our diagonal dictatorship results (Theorems 1 and 4) crucially rely on the admissibility of "linear" or "locally linear" preferences. For such preferences, one may easily imagine how difficult it is to select continuously from the Pareto set. However, no earlier study has shown formally what exactly the cost of continuity is. This paper offers an answer. To attain continuity, we have to pay the cost of diagonal dictatorship, which seems to be too high. 
The diagonal dictatorship result does not hold on domains consisting of only strictly convex preferences. For example, on the domain of Cobb-Douglas preferences, the "Walrasian rule", which is the rule selecting always the unique Walrasian equilibrium allocation, is efficient and continuous. Then, one may well wonder whether efficiency, continuity, and "fairness" are compatible on the full domain of continuous, strictly monotonic, and strictly convex preferences. The answer is left for future study.

2 . In the $n$-agent exchange economies, we showed that only dictatorial rules are efficient, continuous, and strategy-proof over the linear domain and also over the 2-good homothetic domain. In proving these results, we use the diagonal dictatorship feature of efficient and continuous rules, which is a consequence of allowing linear or "locally linear" preferences. In this sense, our proof is not robust. However, we think that even if only strictly convex preferences are admissible, the same impossibility will apply. This is a natural conjecture following from the well-known conjecture by Zhou (1991) and Kato and Ohseto (2001) that the range of every efficient and strategy-proof rule contains only extreme allocations. Let us call this property the "extreme range property".

3. Recently, an important contribution is made by Serizawa and Weymark (2002). They showed that any efficient and strategy-proof rule "cannot guarantee everyone a consumption bundle bounded away from the origin", violating the condition of "minimum consumption guarantee". This means that at least one agent receives bundles sufficiently close to the zero bundle, which is an obnoxious feature. However, it is less obnoxious than (diagonal) dictatorship or the extreme range property. This is because violation of minimum consumption guarantee does not exclude the possibility of choosing only non-extreme bundles. Thus, their result still leaves a substantial gap between "no minimum consumption guarantee" and the extreme range property, conjectured by Zhou (1991) and Kato and Ohseto (2001). Adding continuity, their result implies that at least one agent should receive the zero bundle at some economies, which is still far from (diagonal) dictatorship or the extreme range property.

It is remarkable to note that the proof in Serizawa and Weymark (2002) is quite robust and does not rely on the admissibility of some non-standard or artificial preferences, such as linear preferences or locally linear homothetic preferences that are used in our proofs.

4. The homothetic preference domain $\mathcal{R}_{H}^{N}$ does not include preferences whose indifference curves do not intersect with all the axes, such as Cobb-Douglas preferences. However, these preferences are limit points of $\mathcal{R}_{H}$ (that is, there are 
sequences of preferences in $\mathcal{R}_{H}$, which converge to such preferences). Hence our results can be extended over the larger domain containing $\mathcal{R}_{H}$ and including such preferences.

\section{A Topological properties of the homothetic do- main in the 2-good case}

In this section, we sketch the proofs of the following facts on the homothetic domain with two goods.

Fact 1. $\mathcal{R}_{H}$ is path-connected.

Proof. Let $R_{0}, R_{0}^{\prime} \in \mathcal{R}_{H}$. For all $x \in[0,1]$, let $f(x) \in \mathbb{R}_{+}$satisfy $(x, f(x)) I_{0}$ $(1,0)$. Then the function $f:[0,1] \rightarrow \mathbb{R}_{+}$is well-defined and its graph is the indifference curve of $R_{0}$ through $(1,0)$. Since $R_{0}$ is strictly monotonic and convex, $f$ is monotone decreasing and convex. Since $R_{0}$ is homothetic, the function $f$ completely determines $R_{0}$. Hence we may say that $f$ represents $R_{0}$. Similarly there exists a monotone decreasing and convex function $f^{\prime}:[0,1] \rightarrow \mathbb{R}_{+}$that represents $R_{0}^{\prime}$. For all $\lambda \in[0,1]$, let $f^{\lambda}$ be defined by $f^{\lambda} \equiv \lambda f+(1-\lambda) f^{\prime}$. Then clearly, $f^{\lambda}$ is also monotone decreasing and convex and so there exists a preference $R_{0}^{\lambda} \in \mathcal{R}_{H}$ represented by $f^{\lambda}$. Define $\pi:[0,1] \rightarrow \mathcal{R}_{H}$ as follows: for all $\lambda \in[0,1]$, $\pi(\lambda) \equiv R_{0}^{\lambda}$. It is easy to show that $\pi$ is a continuous path from $R_{0}$ to $R_{0}^{\prime}$.

Fact 2. For all $\varepsilon>0, \mathcal{R}_{H, \varepsilon}$ is path-connected.

Proof. For all $R_{0} \in \mathcal{R}_{H, \varepsilon}$, there exists a strictly convex preference $\bar{R}_{0} \in \mathcal{R}_{H}$ that coincides with $R_{0}$ on $\mathbb{R}_{++}^{2} \backslash C^{\varepsilon}$. Let $R_{0}, R_{0}^{\prime} \in \mathcal{R}_{H, \varepsilon}$. Let $\bar{R}_{0}, \bar{R}_{0}^{\prime} \in \mathcal{R}_{H}$ be the strictly convex preferences that coincide with $R_{0}, R_{0}^{\prime}$ on $\mathbb{R}_{++}^{2} \backslash C^{\varepsilon}$, respectively. Then we can define a continuous path $\pi:[0,1] \rightarrow \mathcal{R}_{H}$ from $\bar{R}_{0}$ to $\bar{R}_{0}^{\prime}$ as in the proof of Fact 1. For all $\lambda \in[0,1]$, there exists $R_{0}^{\lambda} \in \mathcal{R}_{H, \varepsilon}$ such that $R_{0}^{\lambda}$ coincides with $\pi(\lambda)$ on $\mathbb{R}_{++}^{2} \backslash C^{\varepsilon}$. For all $\lambda \in[0,1]$, let $\pi^{\varepsilon}(\lambda) \equiv R_{0}^{\lambda}$. Then it is easy to show that $\pi^{\varepsilon}:[0,1] \rightarrow \mathcal{R}_{H, \varepsilon}$ is a continuous path from $R_{0}$ to $R_{0}^{\prime}$.

Fact 3. $\cup_{\varepsilon>0} \mathcal{R}_{H}^{\varepsilon}$ is dense in $\mathcal{R}_{H}$.

Proof. Let $R_{0} \in \mathcal{R}_{H}$ be strictly convex. For all $n \in \mathbb{N}$, let $R_{0}^{n} \in \mathcal{R}_{H, 1 / n}$ be such that $R_{0}^{n}$ coincides with $R_{0}$ over $\mathbb{R}_{+}^{l} \backslash C^{1 / n}$. It is clear that $R_{0}^{n}$ converges to $R_{0}$ as $n$ goes to infinity. Therefore for each strictly convex preference $R_{0}$ in $\mathcal{R}_{H}$, there exists a sequence of preferences in $\cup_{\varepsilon>0} \mathcal{R}_{H, \varepsilon}$ that converges to $R_{0}$. 
On the other hand, as we show below, the class of strictly convex preferences is dense in $\mathcal{R}_{H}$.

Let $R_{0} \in \mathcal{R}_{H}$ be convex. Pick a strictly convex preference relation $R_{0}^{\prime} \in \mathcal{R}_{H}$. Let $\pi:[0,1] \rightarrow \mathcal{R}_{H}$ be the continuous path from $R_{0}^{\prime}$ to $R_{0}$ defined in the proof of Fact 1. It is clear by definition that for all $\lambda \in(0,1), \pi(\lambda)$ is a strictly convex preference relation in $\mathcal{R}_{H}$. Let $R_{0}^{n} \equiv \pi(1 / n)$. Then $\left(R_{0}^{n}\right)_{n \in \mathbb{N}}$ is a sequence of strictly convex preference relations that converges to $R_{0}$.

Therefore $\cup_{\varepsilon>0} \mathcal{R}_{H, \varepsilon}$ is dense in $\mathcal{R}_{H}$.

\section{References}

[1] Barberà, S. (2001), "An introduction to strategy-proof social choice functions", Social Choice and Welfare 18:619-653

[2] Barberà, S. and Jackson, M. (1995), "Strategy-proof exchange", Econometrica $63: 51-87$

[3] Chichilnisky, G. (1979), "On fixed point theorems and social choice paradoxes", Economics Letters 3:347-351

[4] Chichilnisky, G. (1980), "Social choice and the topology of spaces of preferences", Adv Math 37:165-176

[5] Chichilnisky, G. (1982), "Social aggregation rules and continuity", Quarterly Journal of Economics 96:337-352

[6] Chichilnisky, G. and G.M. Heal (1983), "Necessary and sufficient conditions for a resolution of the social choice paradox", Journal of Economic Theory 31:68-87

[7] Chipman, J.S. (1974), "Homothetic preferences and aggregation", Journal of Economic Theory 8:26-38

[8] Chipman, J.S. and J.C. Moore (1973), "Aggregate demand, real national income, and the compensation principle", International Economic Review 14:153-181

[9] Dasgupta, P., Hammond, P., and Maskin, E. (1979), "The implementation of social choice rules", Review of Economic Studies 46:153-170.

[10] Foley, D. (1967), "Resource Allocation and the Public Sector", Yale Economic Essays 7:45-98 
[11] Gibbard, A. (1973), "Manipulation of voting scheme: a general result", Econometrica 41:587-602

[12] Hurwicz, L. (1972), "On informationally decentralized systems," in McGuire and Radner (eds) Decision and Organization, North-Holland, Amsterdam, pp. 297-336

[13] Hurwicz, L. and M. Walker (1990), "On the generic non-optimality of dominant strategy allocation mechanisms", Econometrica 58:683-704

[14] Ju, B.-G. (2002), "Strategy-proofness versus efficiency in exchange economies: general domain properties and applications", forthcoming in Social Choice and Welfare

[15] Kannai, Y. (1970), "Continuity properties of the core of a market", Econometrica 38(6):791-815

[16] Kato, M. and S. Ohseto (2001), "Toward general impossibility theorems in pure exchange economies", forthcoming in Social Choice and Welfare

[17] Mas-Colell, A., M.D. Whinston, and J.R. Green (1995), Microeconomic Theory, New York: Oxford University Press

[18] Moulin, H. (1980), "On strategy-proofness and single-peakedness", Public Choice 35:435-455

[19] Postlewaite, A. and D. Wettstein (1989), "Feasible and continuous implementation", Review of Economic Studies 56:604-611

[20] Satterthwaite, M. (1975), "Strategy-proofness and Arrow's conditions: existence and correspondence theorems for voting procedures and social welfare functions", Journal of Economic Theory 10:187-217

[21] Satterthwaite, M. and H. Sonnenschein (1981), "Strategy-proof allocation mechanisms at differentiable points", Review of Economic Studies 48:587597

[22] Schummer, J. (1997), "Strategy-proofness versus efficiency on restricted domains of exchange economies", Social Choice and Welfare 14:47-56

[23] Serizawa, S. (2000a), "Inefficiency of strategy-proof rules for pure exchange economies", forthcoming in Journal of Economic Theory 
[24] Serizawa, S. (2000b), "Pairwise strategy-proofness", Working Paper, Tohoku University.

[25] Serizawa, S. and J.A. Weymark (2002), "Efficient strategy-proof exchange and minimum consumption guarantees", Vanderbilt University, Working Paper No.02-W16.

[26] Sprumont, Y. (1991), "The division problem with single-peaked preferences: a characterization of the uniform rules", Econometrica 59:509-519

[27] Sprumont, Y. (1995a), "Strategy-proof collective choice in economic and political environments", Canadian Journal of Economics 28:68-107

[28] Sprumont, Y. (1995b), "A note on strategy-proofness in Edgeworth-Box economies", Economic Letters 49:45-50

[29] Thomson, W. (1984), "The manipulability of resource allocation mechanisms", Review of Economic Studies LI:447-460

[30] Thomson, W. (1995), The Theory of Fair Allocation, manuscript, University of Rochester

[31] Thomson, W. (2001), "Strategy-proof allocation rules on economic domains", University of Rochester, mimeo

[32] Zhou, L. (1991), "Inefficiency of strategy-proof allocation mechanisms in pure exchange economies", Social Choice and Welfare 8:247-254

[33] Zhou, Y. (1997), "A note on continuous social choice", Social Choice and Welfare 14:245-248 